\title{
Regionale Ressourcen erschließen
}

\section{Das Bundesprogramm »Kompetenzagenturen « am Beispiel des Landkreises Saalfeld-Rudolstadt}

\author{
Kerstin Carol
}

Kerstin Carol ist Leiterin der

Kompetenzagentur des Landkreises

Saalfeld-Rudolstadt.

E-Mail k.carol@bz-saalfeld.de
Das Programm »Kompetenzagenturen " des Bundesministeriums für Familie, Senioren, Frauen und Jugend richtet sich an die Jugendlichen und jungen Menschen, die von sich aus den Zugang zu den Unterstützungsangeboten nicht finden oder von den Systemen im Übergang zwischen Schule und Beruf nicht profitieren.

Um besonders benachteiligten Jugendlichen angemessene Hilfen beim Zugang zu Ausbildung und Arbeit geben zu können, beschloss das Bundesministerium für Familie, Senioren, Frauen und Jugend im Jahr 2002 das Modellprogramm »Arbeitsweltbezogene Jugendsozialarbeit«. Im Rahmen dieses Programms wurde die Modellphase "Kompetenzagenturen « in 15 deutschen Städten gestartet. Als Ergebnis der erfolgreichen Pilotphase erschien 2006 eine neue Förderrichtlinie zur Durchführungsphase, in der eine Ausweitung der Kompetenzagenturen auf bundesweit 200 Standorte festgelegt wurde.

Die Angebotsformen und Strukturen der Benachteiligtenförderung sowie der Jugendsozialarbeit sind sowohl in den jeweiligen Bundesländern als auch in den einzelnen Landkreisen und Regionen verschieden ausgeprägt, sodass sich für die jeweiligen Kompetenzagenturen regional unterschiedliche Anforderungen an die Schwerpunkte, Angebote und Strukturen ergeben. Entsprechend dem spezifisch ermittelten, regionalen Bedarf richten sich die einzelnen Kompetenzagenturen an unterschiedliche Personengruppen. Bedarfsorientiert kann präventive oder intervenierende Jugendsozialarbeit fokussiert werden.

In diesem Programm werden ausdrücklich besonders benachteiligte Jugendliche und junge Menschen zwischen 14 und 27 Jahren erfasst, die von den bestehenden Angeboten nicht profitieren. Soziale Benachteiligungen erwachsen aus einer defizitären Sozialisation der jungen Generation und können in einer misslingenden gesellschaftlichen Integration gipfeln. So können beispielsweise Schulmüdigkeit, Delinquenz und Hafterfahrungen, Verschuldung, Obdachlosigkeit, Migrationshintergrund und familiäre Schwierigkeiten einen ungehemmten Eintritt in Ausbildung und Beruf behindern. Auch individuelle Beeinträchtigungen wie etwa Lernund Leistungsschwächen, motorische und sprachliche Entwicklungsstörungen, Behinderungen, Persönlichkeitsstörungen oder Suchtkrankheiten jeglicher Art können sich negativ auf die berufliche und soziale Integration auswirken.

Jugendliche, die von bestehenden Hilfsangeboten beim Übergang von der Schule in die Ausbildung nicht profitieren (können), werden von den Kompetenzagenturen gezielt aufgesucht und zurück auf ihren Weg in den Beruf gebracht. Hilfebedürftige, die nicht selbstständig oder durch Familie, Freunde und Bekannte den Weg zur Kompetenzagentur finden, werden von den Mitarbeitern der Jugendund Schulämter, Agenturen für Arbeit und Arbeitsgemeinschaften an die Kompetenzagenturen vermittelt.

Basis einer professionellen Zusammenarbeit des Case Managers mit dem Jugendlichen ist das Prinzip der Freiwilligkeit. Dadurch können negative Auswirkungen von Beratungen in Zwangskontexten ausgeräumt und eine bessere, symmetrische Arbeitsbeziehung hergestellt werden. Diese stellt eine unabdingbare Voraussetzung für eine stetige Motivation und Kooperationsbereitschaft des Jugendlichen dar, um eine selbstständige und eigenverantwortliche Lebensführung zu erreichen.

Mithilfe des individuellen Instruments des Case Managements wird den Jugendlichen eine Palette an individuell und passgenau abgestimmten Unterstützungen angeboten, die über die Grenzen bisheriger Einzelmaßnahmen hinausgehen. Hierbei nehmen die Mitarbeiter der Kompetenzagenturen eine, dem komplexen Förderbedarf angemessene, Beratungs-, Vermittlungs- und Lotsenfunk- 
tion wahr. Unter Einbeziehung entsprechender Fachstellen wird durch den Case Managementprozess ein zielgerichteter und schrittweiser Abbau der persönlichen Hemmnisse unterstützt.

Zur Leistungssteuerung sind lokale Netzwerke unabdingbar. Eine kontinuierliche Fallarbeit braucht gefestigte Netzwerkpartner, die gemeinsam und kooperativ auf individuelle Problemlagen der jungen Menschen reagieren können.

\section{Beispiel}

In der Durchführungsphase des Programms des Bundesministeriums für Familie, Senioren, Frauen und Jugend wurde am 1. November 2006 die Kompetenzagentur des Landkreises SaalfeldRudolstadt gegründet. Die Konzeption berücksichtigt die landkreisspezifische Infrastruktur, Bildungsmöglichkeiten und bezieht die bestehenden Netzwerke ein.

Thüringenweit gehören 23 Prozent der jugendlichen Arbeitslosen zur Personengruppe der Langzeitarbeitslosen und 12 bis 15 Prozent gelten wegen besonderer Hemmnisse, die einen reibungslosen Inte- grationsprozess behindern, als schwer vermittelbar. (vgl. TMSFG, 2007) Für die Jugendlichen im Landkreis Saalfeld-Rudolstadt kommt die typisch ländliche Strukturierung beim Zugang zu Bildungsund Hilfsangeboten erschwerend hinzu. Diese regionalen Spezifika bilden die Basis für das Handlungsprofil der Agentur.

Der Träger der Kompetenzagentur des Landkreises Saalfeld-Rudolstadt ist die Bildungszentrum Saalfeld GmbH in Kooperation mit der Jugendberatung der Evangelischen Stiftung Christopherushof.

\section{Prävention}

Der Übergang von der Schule zur Ausbildung stellt für junge Menschen einen bedeutsamen Abschnitt im Prozess der beruflichen Entwicklung dar. Dies gilt insbesondere für Jugendliche mit einem erhöhten Förderbedarf. Aus diesem Grund richtet sich der Fokus der Kompetenzagentur Saalfeld-Rudolstadt an die Übergänge an den Schwellen zwischen Schule und Ausbildung bzw. Ausbildung/Maßnahme und Beruf, an denen die Integration Jugendlicher gefährdet ist.
Ziel ist es, diese Übergänge reibungslos zu gestalten und möglichst wenig Jugendliche an den Übergängen $\mathrm{zu}$ »verlieren «. Um dies zu gewährleisten, setzt die Arbeit der Kompetenzagentur nicht erst an, wenn die Jugendlichen diese Schnittstellen erreichen, sondern bereits in den Vorabgangsklassen der allgemeinbildenden Schulen des Landkreises.

\section{Intervention und Integration}

Neben der Schwerpunktsetzung auf präventive Arbeit hält auch die Kompetenzagentur Saalfeld-Rudolstadt am Konzept der Integration besonders benachteiligter Jugendlicher fest. Dabei richtet die Kompetenzagentur ihre Unterstützung an den spezifischen Multiproblemlagen der Jugendlichen aus und verfolgt einen individuellen Unterstützungs- und Förderansatz. Durch aufsuchende Arbeit wird ein niedrigschwelliger Zugang $\mathrm{zu}$ den Jugendlichen gefunden, die von den vorhandenen Bildungs-, Ausbildungs- und Beschäftigungsangeboten nicht erreicht werden oder sich diesen entziehen (vgl. BMFSFJ, 2002, 32).

\section{Fallbeispiel Hannes, 17 Jahre}

- deutsch

- ledig, feste Freundin

- Eltern geschieden, lebt beim Vater

- Bedarfsgemeinschaft ALG II

Schulausbildung:

- 9 Jahre Realschule

- Hauptschulabschluss

Vermittlungshemmnisse:

- mangelnde Lerneinstellung (Schulmüdigkeit)

- aus überbetrieblicher Erstausbildung gekündigt

- Schulden

- mangelnde Sozialkompetenz

- gestörte Familienverhältnisse

Kompetenzen:

- Schulabschluss

- feste Bindung zur Freundin

- Vorhandensein beruflicher Ziele

- Motivationsbereitschaft

Chancen:

- freiwillige Annahme des Unterstützungsangebotes der Kompetenzagentur

- motiviert und änderungsbereit

- Unterstützung durch die Freundin

Begleitung durch die Kompetenzagentur:

- seit April 2007
Ziele:

- berufliche Ausbildung ab September 2007

- Stabilisierung der Sozialkompetenz

- Schuldenabbau

Integrationsstufen:

1. Information durch Sozialpädagoge des Bildungsträgers seiner Erstausbildung

2. Kontaktaufnahme zu Hannes im Jugendzentrum, Angebot des Case Managements

3. Gespräche im sozialen Umfeld (Vater, ehemalige Klassenleiterin, Freundin, Jugendzentrum etc.)

4. Kontaktaufnahme zur ARGE und Unterstützung bei Antragstellung

5. Vermittlung des Kontaktes zur Schuldnerberatung

6. Beratung zur Wohnsituation und Unterstützung bei der Wohnungssuche

7. Feststellen der beruflichen Kompetenzen (Hamet-Verfahren)

8. Begleitung zur Berufsberatung

9. Teilnahme an Trainingsmaßnahme bis August 2007

10. Anmeldung im Sportverein

\section{Perspektiven:}

- Aufnahme einer betrieblichen Ausbildung zum Koch

- regelmäßige Treffen mit Hannes und seiner Freundin

- weitere Begleitung des Schuldenabbaus

- regelmäßige Information seines Vaters 
Hierfür werden bei den Partnern vor Ort (z. B. Jugendzentren, Schulen, soziale Einrichtungen der Jugendhilfe, jugendtypische Lokale, usw.) Beratungstermine ermöglicht. Durch eine zeitlich nicht determinierte Begleitung durch Case $\mathrm{Ma}$ nagement wird dem Jugendlichen eine auf seine individuellen Voraussetzungen und Entwicklungen ausgerichtete Unterstützung geboten, in deren Zentrum das Heranführen an eine selbstständige Lebensweise steht (vgl. Kasten »Fallbeispiel Hannes, 17 Jahre ).

\section{Koordination und Netzwerk}

Netzwerke stellen die wesentliche Ressource des Case Managements dar. Im Landkreis Saalfeld-Rudolstadt existiert ein breites Spektrum an Bildungsund Hilfsangeboten sowie vereinzelte Netzwerke der Jugendhilfe. Als Koordinationsinstanz ist es Aufgabe der Kompetenzagentur, diese bereits vorhandenen Angebote zu einem professionellen Helfernetzwerk zusammen zu führen.

Aufgrund der Rahmenbedingungen werden bestehende Netzwerkstrukturen teilweise erweitert oder vorhandene Kooperationsbeziehungen neu formiert. Etwaige Lücken im Hilfssystem der Region werden aufgedeckt und durch die Initiierung neuer Angebote geschlossen. Eine dadurch bewirkte Verbesserung der Kommunikationsstruktur schließt Informationslücken zwischen den Netzwerkpartnern und beugt ineffizienten Maßnahmezuweisungen vor.

Zur Begleitung des Fortschritts und Reflexion der Ergebnisse wurde ein Projektbeirat installiert, der sich aus den Geschäftsführern und Amtsträgern, der am Integrationsprozess der Jugendlichen beteiligten Institutionen, zusammensetzt. Somit bleibt die Transparenz der Jugendsozialarbeit ebenso gewährleistet wie der Informationsaustausch über aktuelle Bildungsangebote in der Region. Im Falle einer mangelnden Präsenz an Maßnahmemöglichkeiten können neue Konzeptionen angeregt und entwickelt werden, die die berufliche und soziale Integration der Zielgruppe begünstigen.

\section{Fazit}

Während der bisherigen Laufzeit der Kompetenzagentur im Landkreis Saalfeld-Rudolstadt wurde deutlich, dass mit dem Programm »Kompetenzagenturen « des Bundesministeriums für Familie, Senioren, Frauen und Jugend ein flexibles, einzelfallorientiertes und neutrales Instrument geschaffen wurde, das an die Bedarfe der Region anknüpft und eine individuelle Einzelfallhilfe für den Jugendlichen sowie die Vernetzung der Benachteiligtenförderung ermöglicht.

Durch die Aktivierung klienteneigener Ressourcen und eine effektive und zielgerichtete Ausnutzung von Hilfsangeboten bietet das Programm langfristig eine Steigerung der wirtschaftlichen Effizienz des Landkreises.

\section{Literatur}

Thüringer Ministerium für Soziales, Familie und Gesundheit (TMSFG): Regionaltreffen der Thüringer Kompetenzagenturen, Präsentation vom 29. März 2007, Erfurt, 2007.

Kompetenzagenturen. Neue Modellphase im Bundesmodellprogramm »Arbeitsweltbezogene Jugendsozialarbeit « in: Bundesministerium für Familie, Senioren, Frauen und Jugend (BMFSFJ) (Hg.): Rundbrief Ausbildung Nr.1, 2002.

Institut für berufliche Bildung, Arbeitsmarkt- und Sozialpolitik GmbH (Hg.): Modellprogramm Arbeitsweltbezogene Jugendsozialarbeit. Modellphase: Kompetenzagenturen. Aus der Praxis des Modellprogramms. Gebündelte Kompetenzen für berufliche Integration. Werkstattbericht Juli 2006, Offenbach am Main, 2006.

\section{Anwalt für benachteiligte Jugendliche: der Kooperationsverbund Jugendsozialarbeit}

Kompetenz bündeln, Fachlichkeit konsequent weiter entwickeln und qualifizierte Politikberatung - das sind die wesentlichen Aufgaben des Kooperationsverbundes Jugendsozialarbeit. Dieser aus sieben Bundesorganisationen und Spitzenverbänden bestehende Zusammenschluss nahm am 1. Juli 2007 seine Tätigkeit auf. Handlungsprinzip ist arbeitsteiliges und kooperatives Vorgehen. Ein in Berlin eingerichtetes Büro koordiniert die Aktivitäten und die Öffentlichkeitsarbeit. Für die fachliche Weiterentwicklung wurden Federführungsfunktionen verteilt:

- Die Arbeiterwohlfahrt bearbeitet die Felder Bildung und Bildungspolitik sowie Jugendsozialarbeit an der Schnittstelle zu anderen Leistungen der Jugendhilfe.

- Die Bundesarbeitsgemeinschaft der Evangelischen Jugendsozialarbeit hat sich europäischen Themen und Förderprogrammen sowie dem Aufgabenfeld der Förderung junger Migrantinnen und Migranten verpflichtet.

- Den Handlungsschwerpunkt Jugendwohnen bringt die Bundesarbeitsgemeinschaft der Katholischen Jugendsozialarbeit ein.

- Die Bundesarbeitsgemeinschaft der örtlich regionalen Träger der Jugendsozialarbeit engagiert sich in der beruflichen Integrationsförderung und Jugendberufshilfe sowie bei der Gestaltung des Übergangsmanagements an den Schnittstellen der Jugendberufshilfe.

- Der Paritätische Gesamtverband befasst sich mit dem Schwerpunkt Arbeitsmarktpolitik und Jugendsozialarbeit.

- Mit dem Themenkomplex Jugendarbeit und Schule sowie Fragen der Partizipation junger Menschen beschäftigt sich das Deutsche Rote Kreuz.

- Der Internationale Bund transportiert die Themen berufliche Integrationsförderung, politische Bildung im Rahmen der Jugendsozialarbeit sowie den internationalen Jugendaustausch.

Die sieben Bundesorganisationen sind die wesentlichen Akteure der Jugendsozialarbeit in Deutschland. Sie übernehmen anwaltliche Funktionen für benachteiligte Jugendliche und sensibilisieren die Öffentlichkeit für ihre Lebenslagen. Als Kooperationsverbund beobachten sie die fachlichen Entwicklungen, geben Impulse und schaffen eine Plattform zur Weiterentwicklung der Jugendsozialarbeit in Deutschland.

Kooperationsverbund Jugendsozialarbeit, Chausseestraße 128/129, 10115 Berlin, Telefon 030 288789-38, Fax 030-288 78955 , E-Mail kooperationsverbund@jugendsozialarbeit.de 